American Journal of Internal Medicine
$2021 ; 9(1): 26-35$
http://www.sciencepublishinggroup.com/j/ajim
doi: $10.11648 /$ jajim.20210901.15
ISSN: $2330-4316$ (Print); ISSN: $2330-4324$ (Online)

\title{
Multi-organ Dysfunction Due to SARS-CoV-2 Infection: A Comparative Overview
}

\author{
Moshiul Alam Mishu*, Fairoz Samiha, Kohinoor Zahan, Akash Saha, Shahida Ferdousee \\ Department of Biochemistry and Molecular Biology, Shahjalal University of Science and Technology, Sylhet, Bangladesh \\ Email address: \\ moshiul-bmb@sust.edu (M. A. Mishu), samiha1998@student.sust.edu (F. Samiha), kohinoor035@student.sust.edu (K. Zahan), \\ akash036@student.sust.edu (A. Saha), ferdousee.2017@student.sust.edu (S. Ferdousee) \\ *Corresponding author
}

\section{To cite this article:}

Moshiul Alam Mishu, Fairoz Samiha, Kohinoor Zahan, Akash Saha, Shahida Ferdousee. Multi-organ Dysfunction Due to SARS-CoV-2 Infection: A Comparative Overview. American Journal of Internal Medicine. Vol. 9, No. 1, 2021, pp. 26-35.

doi: 10.11648/j.ajim.20210901.15

Received: November 26, 2020; Accepted: December 15, 2020; Published: January 22, 2021

\begin{abstract}
SARS-CoV-2 or COVID-19 is a highly transmittable and pathogenic viral disease, which was first reported in the province Wuhan of China in late December 2019. The disease has shown its catastrophic effect in more than 200 countries and territories, causing more than 53.7 million confirmed cases and over 1.3 million deaths since its outbreak. Although the virus was primarily presumed to cause respiratory disease, reports are emerging of the plausible impact of the virus on multiple organs. The multi-organ dysfunction in severe patients of COVID-19 results in high morbidity and mortality rate compared to the other coronavirus family members like SARS and MERS. In this review, we provided a brief overview of the current insights of potential COVID-19 impacts on multiple organ functions. Reports suggest that the virus may exert direct (e.g., using ACE2 receptor) or indirect (e.g., cytokine storm) effects on several organs, including the lung, kidney, heart, liver, and brain. These multi-organ injuries may contribute to poor health outcomes and even may lead to death, especially to those suffering from cardiac disease, diabetes, liver, kidney diseases, etc. However, further investigation is required to know the exact mechanism of the infection. This study will provide valuable information on multi-organ dysfunction due to COVID-19 and thus, help clinicians to combat the disease until an effective vaccine arrives.
\end{abstract}

Keywords: SARS-CoV-2, Pandemic, Multi-Organ Failure, Angiotensin-Converting Enzyme 2 (ACE2), Cytokine Storm

\section{Introduction}

At the end of December 2019, the primary reports of the viral disease stood out when a group of patients with pneumonia was seen in Wuhan, the capital of Hubei, China. Genetic evidence proves that they are hiding out in nature especially in animals, possibly for decades, and obtained the power to infect humans by a genetic mutation. Several characteristics are required to declare a pandemic situation such as a unique type of infection, which covers huge geographical regions, and has excessive attack rates, etc. [1]. Swine flu, Influenza, Spanish flu, Cholera, and Bubonic plague are the major pandemics that occur in previous human history. On February 11, The International Committee on Taxonomy of Viruses has renamed the virus as severe acute respiratory syndrome coronavirus-2 (SARS-CoV-2), and WHO officially named and declared COVID-19 as pandemic later on March 11, 2020 [2]. The National Health Commission of China provides information about the novel coronavirus that had more than $95 \%$ sequence similarities with the bat coronavirus and more than $70 \%$ similarities with the previous SARS-CoV [3]. However, the earlier cases indicated bat as the primary reservoir for the Covid-19 virus emerging from the live animal market which then quickly transmitted via human to human contact [4].

SARS-CoV-2 can bind with the alveolar pneumocytes, where Angiotensin Converting Enzyme 2 (ACE2) is expressed greatly. SARS-CoV-2 can readily attack cells at multiple points of the host (lung and throat being the primary target), which is different from other classes of this virus [5]. Apart from causing pulmonary disease, SARS-CoV-2 has the potentiality to infect other organs of the human body as ACE2 expresses in nearly all the organs. Though it is not clear that how SARS-CoV-2 spread into several organs, 
some predicted pathways have been deducted by researchers. In this context, we are focusing to find out and co-relate the mechanisms by which SARS-CoV-2 infects.

The COVID-19 virus started to show its catastrophic impact on 2020 by emerging globally. In total 53,732,556 confirmed cases were recorded worldwide including $1,308,572$ deaths $(2.44 \%$ of total infected cases) by WHO till $14^{\text {th }}$ November 2020 (Table 1). According to WHO, COVID19 becomes severe to those with a compromised immune system and have other health complications like cardiac disease, diabetes, liver, and kidney diseases. To control the pandemic situation, the WHO provides accurate information and statistics to help countries to prepare and respond against the virus. Despite the low death rate, the fast and rapid transmission of Covid-19 makes it a serious concern for everyone.

\section{Virology}

Coronaviruses are enveloped positive-sense singlestranded RNA viruses ranging from $80-220 \mathrm{~nm}$ in diameter with a crown-like spike which is $20 \mathrm{~nm}$ in diameter [The structure of SARS-CoV-2 has been discussed in Figure 1]. Coronaviruses are members of the subfamily coronavirinae in the family coronaviridae. The subfamily of CoVs is genotypically divided into four genera: $\alpha, \beta, \gamma$, and $\delta$ CoVs. $\alpha$ and $\beta$-CoVs mainly cause infections in humans. SARS-CoV2 as well as SARS-CoV and MERS-CoV are part of these $\beta$ $\mathrm{CoV}$ genera. SARS-CoV-2 has a zoonotic origin and bats are accounted for the best reservoirs for zoonotic viruses. Until now, it stays conflicting concerning the cause of SARS-CoV2, and etiologic examinations are being led by Chinese health authorities. Phylogenetic research indicates that SARS-CoV has $79.5 \%$ and MERS-CoV has a $50 \%$ sequence identity with SARS-CoV-2. Similar to other $\beta$-CoVs, the genome size of SARS-CoV-2 virion is $29.9 \mathrm{~kb}$ and the SARS-CoV-2 S protein has a full size of 1,273 amino acids [6-8]. The nucleotide sequence of spike (S) protein of SARS-CoV-2 (which mainly interacts with host cell receptors) shares $72 \%$ sequence similarity with SARS-CoV. The SARS-CoV-2 virion has a nucleocapsid composed of genomic RNA and phosphorylated nucleocapsid $(\mathrm{N})$ protein. Gene order of SARS-CoV-2 is 5'-replicase ORF1ab-S-envelope (E)membrane-(M)-N-3'. The nucleocapsid is made with phospholipid bilayers and enclosed by two different types of spike proteins: the spike glycoprotein trimmer (S), and the hemagglutinin-esterase (HE). In the viral envelope, the membrane $(\mathrm{M})$ protein and the envelope $(\mathrm{E})$ protein are found within the $\mathrm{S}$ proteins. Almost 15,018 mutations were observed by China National Center for Bioinformation. The $21 \mathrm{~kb}$ sized ORF1ab gene contains 16 predicted nonstructural proteins, several downstream ORF like other SARS-CoV. Feasibly the polybasic (furin) cleavage site insertion at the junction of the S1 and S2 subunit of the S protein increases the infectivity of the virus [9]. Most of these non-structural proteins of SARS-CoV-2 show more than $85 \%$ sequence similarities with SARS-CoV; except for the accessory gene ORF8 of SARS-CoV-2, encoding a novel protein (shows only $40 \%$ sequence similarities). Interestingly, a 382-nucleotide deletion covering the whole ORF8 of SARS-CoV-2 has been discovered during a genomic study in Singapore. This sort of deletion might illustrate how does the virus adapt in humans while transmitting from an animal host [8].

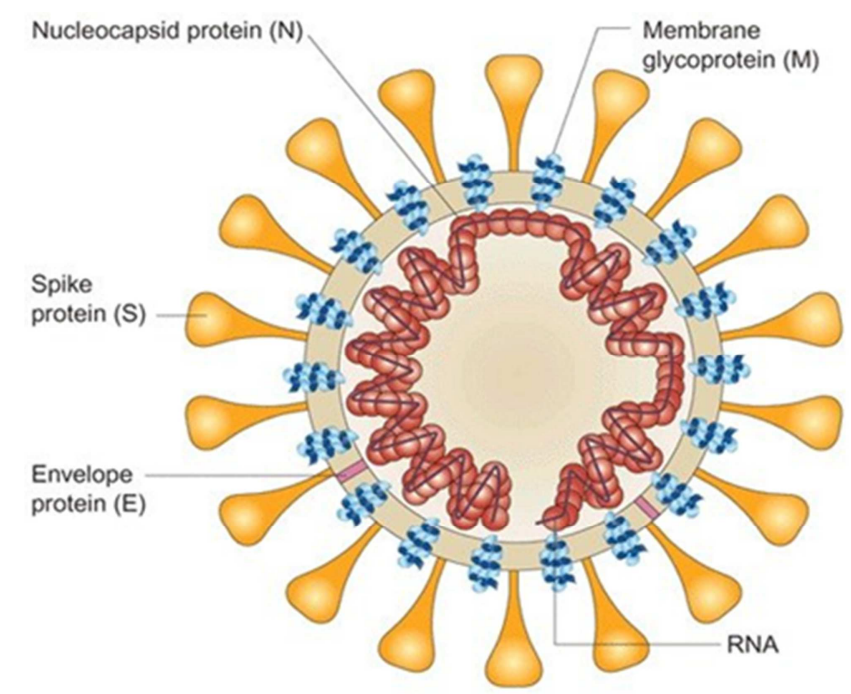

Figure 1. Structure of the SARS-CoV-2. SARS-CoV-2 is an enveloped, positive-sense-single-stranded RNA virus. The viral surface proteins (spike, envelope, and membrane) are immersed in a lipid bilayer envelope derived from the host cell. The nucleocapsid protein covers the ssRNA of the virus [10].

\section{Pathogenicity}

Novel coronavirus can spread via fecal-oral transmission along with respiratory droplets [11]. A significant amount of coronavirus patients experiences severe diarrhea, nausea, vomiting, and abdominal discomfort due to the accumulation of the virus in the lungs and affecting other organs as well. Scientists also find a detectable amount of viral RNA in the fecal samples [12]. Respiratory droplets of an infected person may infect other people coming in contact within a range of three to six feet. According to the type of surface, temperature, and humidity of any environment, coronavirus may persist on a surface from a few hours to several days $[13,14]$. The virus mostly affects the alveolar cells of the respiratory system [15].

The surface of SARS-CoV-2 has a series of spike proteins that look like a crown under an electron microscope and thus give rise to the name corona [6]. The spike proteins allow the entry of the virus into the host cell by attaching to the membrane of the cell through a specific binding receptor called "The Angiotensin-Converting-Enzyme 2 (ACE2)". ACE2 is expressed in the lung, heart, kidney, and intestine and also in the bile duct $[14,15]$. The spike protein of the virus has two subunits in it, a) S1, which mainly binds to the host cell receptor, and b) S2 which mediates the fusion of the viral and cellular membranes. The virus mostly attacks type- 2 pneumocytes through its spike protein via the ACE2 receptor. 
The virus gets into the cell by endocytosis. As soon as it gets into the cell, it releases its ssRNA molecule. Eventually, the RNA molecule takes over the host cell ribosome and start producing different protein molecules of it. It also utilizes the RNA-dependent RNA polymerase enzyme to synthesize its genome RNA. As coronavirus damages type-2 pneumocytes, it can secret numerous cytokines and inflammatory products [16]. This activates alveolar macrophages, which then releases specific Interleukin-1, Interleukin-6, and tumor necrosis factor (TNF). These molecules come into the capillaries surrounding the alveoli and cause capillaries smooth muscle relaxation [17]. They are also responsible for endothelial cell contraction and relaxation. All these events finally lead to vasodilation and increase capillary permeability. Besides, loss of surfactant due to damage to type-2 pneumocytes, resulting in increased surface tension, and eventually, alveoli collapse [18].

The collapsed alveoli may decrease the gas exchange, which will cause hypoxia (a decrease of the oxygen concentration in the blood along with trouble in breathing). Increased inflammatory cytokines will attract neutrophils that can release reactive oxygen species and other proteases to kill the virus or virus-infected cell. At the same time, these oxygen species and proteases can damage the surrounding type 1 and type 2 pneumocytes and thus reducing the gas exchange and mechanical irritation leading to cough. IL-1 and IL- 6 can then be traveled into the hypothalamus and stimulate it to release specific prostaglandins that increase the body temperature resulting in fever [19].

Again, the low partial pressure of oxygen can stimulate the chemoreceptors and these receptors would stimulate the sympathetic system. The heart will try to pump more and more blood to make the oxygen demand and the heart rate will increase consequently. On the other hand, the respiratory rate will also increase as the body need more oxygen to inhale and exhale more carbon dioxide [20, 21]. Mortality rate increases due to COVID-19 with patients suffering from heart disease, asthma, diabetes, chronic respiratory disease, cancer, and hypertension. These conditions make both the innate and adaptive immune systems vulnerable to fight against the disease and may lead to multi-organ failure [14, $15,22]$.

\section{Worldwide Perspective}

As of $14^{\text {th }}$ November 2020 , the WHO reported over 53.7 million confirmed COVID-19 cases including more than 1.3 million deaths worldwide, and 216 countries and territories are affected by the virus (Table 1). Among the reported COVID-19 cases, the Asian continent has a higher recovery rate whereas Europe has a higher number of active cases compared to other continents. Furthermore, case fatality rates (CFR) across continents due to COVID-19 are currently South America (3.0\%), North America (2.9\%), Africa (2.4\%), Oceania (2.4\%), Europe (2.3\%), and Asia (1.8\%) respectively.

Table 1. Worldwide COVID-19 Scenario

\begin{tabular}{|c|c|c|c|c|c|c|}
\hline Continents & Total Cases & Total Deaths & Total Recovered & Active Cases & Total Population & Population Density $/ \mathrm{km}^{2}$ \\
\hline Asia & $14,904,676$ & 263,281 & $13,295,832$ & $1,345,563$ & $\sim 4.6$ Billion & 150 \\
\hline Africa & $1,961,099$ & 46,906 & $1,650,737$ & 263,456 & 1.3 Billion & 45 \\
\hline Europe & $13,484,610$ & 315,654 & $4,977,740$ & $8,191,216$ & 747 Million & 34 \\
\hline North America & $13,067,017$ & 373,630 & $8,291,984$ & $4,401,403$ & 369 Million & 20 \\
\hline Oceania & 42,088 & 994 & 32,882 & 8,212 & ４2 Million & 5 \\
\hline South America & $10,272,345$ & 308,092 & $9,264,333$ & 699,920 & $\sim 655$ Million & 32 \\
\hline In Total & $53,732,556$ & $1,308,572$ & $37,514,167$ & $14,909,817$ & & \\
\hline
\end{tabular}

Source: WHO, Worldometer (https://www.worldometers.info/coronavirus/); Last Updated: 14/11/2020 at 11:59 pm

The actual number of infected cases is suspected to be higher than documented and expected to be nearly $10 \%$ of the world's population. The possible reasons behind undocumented reports might be asymptomatic infections that are not tested, a limited number of testing facilities, particularly in developing countries. Apart from the differences in mortality rates, the countries and territories can be attributed to considerable heterogeneity in virus dissemination, variability in infection fatality rate (IFR), and erroneous counting of deaths [23]. Besides, risk factors for severe COVID-19 are considered to be old age and underlying health issues, such as cardiovascular disease, diabetes, chronic kidney disease, etc., which may differ in population across different countries [24, 23]. In a recent study, Clark et al. (2020) suggested that about 1.7 (range 1.02.4) billion people, $22 \%$ (range 15-28) of the global population, have at least one underlying disease that could increase their risk to COVID-19 [24]. Although this is a massive figure, the consequence would likely ensue if substantial interventions such as effective and safe vaccines are not available faster for preventing the further spread of the infection [23].

While some countries worldwide have succeeded in the containment of the epidemic, others are still dealing with the first wave with the steady rise and decline of regular cases and deaths [25]. Epidemiologists indicated that a stronger second wave of COVID-19 pandemic is likely to occur before the end of 2020. Future waves of the pandemic may vary geographically, and their severity is likely to depend on the efficacy of local control policies [23]. Researchers are speculating that the COVID-19 pandemic may exist for a few more years and maybe slowed down if the majority of the world's population (more than 60\%) develops herd immunity from the infection either naturally or by vaccination [26, 27]. The herd immunity threshold depends mainly on the basic reproduction number (R0) of an infection [27]. Although the 
exact R0 value for COVID-19 is very hard to determine, as many asymptomatic cases were not accounted for at this stage. An estimated R0 value of 2.5 (range: 1.8-3.6) is suggested for SARS-CoV-2 recently, which was quite similar compared to the R0 value (range: 2.0-3.0) of SARS-CoV [8]. Considering the estimated value of R0 for SARS-CoV-2, the threshold for herd immunity would be approximately $60 \%$; which implies that to achieve herd immunity, about $60 \%$ of the world's population must be infected with the virus [23, 27]. Without vaccination, achieving herd immunity naturally is considered theoretically possible if the rapidly evolving infection tends to span a few more years and is not strictly controlled [27]. However, this would be catastrophic as millions would succumb to the disease, and many susceptible individuals will die [23]. Therefore, for long-term prevention and control of this current pandemic and its future spread, effective and safe vaccinations are the most efficient method, and widespread vaccinations of the population globally, particularly of the susceptible individuals, should be conducted faster. To date, no commercially approved vaccines for COVID-19 have been available, although certain vaccines are in phase II human clinical trials and others have progressed to phase III (e.g., Sputnik V, ChAdOx1, CanSino, etc.) $[8]$.

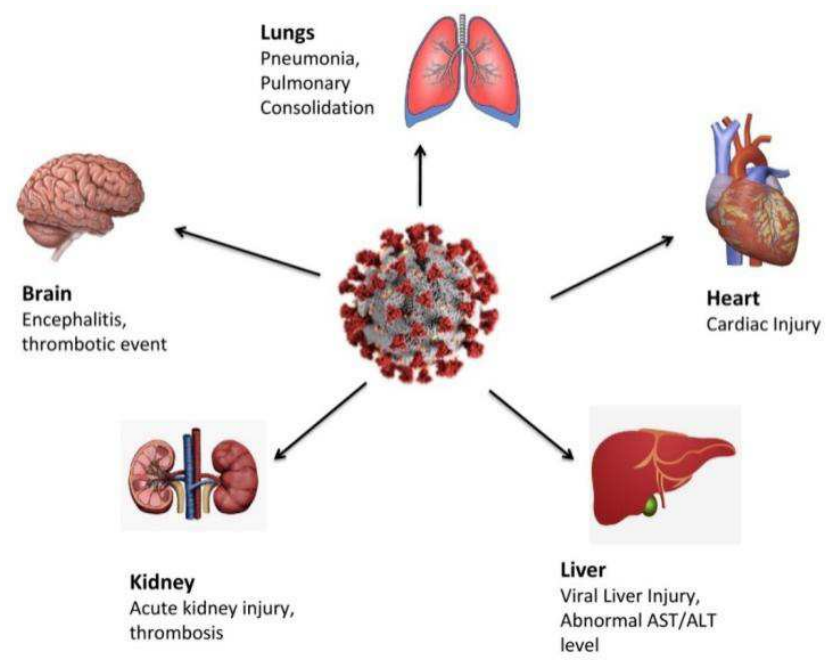

Figure 2. Graphical representation of the impact of SARS-CoV-2 on different organs. Studies suggested that Lung, Heart, Liver, Brain, Kidney, etc. could be damaged due to the infection of SARS-CoV-2.

\section{Multi-organ Dysfunction by SARS-CoV-2}

\subsection{Lung Injury}

Researchers divided human COVID-19 into two categories. Low pathogenic COVID-19 infects the upper respiratory tract and Highly pathogenic COVID-19 can infect the lower respiratory tract causing severe acute respiratory syndrome [28]. In most COVID-19 patients, computed tomography (CT) scans demonstrate ground-glass opacity (GGO), crazy-paving pattern, consolidation, and traction bronchiectasis, and in some observations, pleural effusion and lymphadenopathy were found [29-32]. Continuous CT image follow-up in affected patients indicated that COVID19 pneumonia in the lung gradually develops from focal unilateral to diffuse bilateral ground-glass opacification, predominantly in the lower lobes, and which may contribute to the further progression of crazy-paving pattern and subsequent consolidation [29, 31]. Being highly opportunistic pathogens, COVID-19 may attack the lower respiratory tract and result in severe complications, like pneumonia, exacerbations of asthma, bronchitis, bronchiolitis, and respiratory distress syndrome [33]. Besides, in the hospital settings, patients can be categorized into two distinct phenotypes respectively "Type L" and "Type H", considering the lung mechanics and respiratory distress as well as monitoring CT scans. "Type L" patients can be identified by poor lung elasticity, decreased lung weight, and a low response to positive end-expiratory pressure (PEEP) and may not specifically have dyspnea. The "type H" patients, by contrast, demonstrate considerable consolidation of CT, high lung elasticity, increased lung weight, and high PEEP responses, which display the properties of acute respiratory distress syndrome (ARDS). These conceptual methods might help to comprehend the severity and extent of COVID-19, and also the management of different therapeutic interventions and ventilatory strategies depending on the underlying physiology to alter the outcome of patients [34, 35]. Furthermore, histopathology of the lungs revealed predominant exudative and proliferative diffuse alveolar damage (DAD) including widespread capillary congestion, discrete hyaline membranes, pneumocytes depletion, edema, and pulmonary embolism $[36,37]$.

Severe lung injury can be caused by two reasons: One, viral infection, and another is immune overactivation. COVID-19 binds with the ACE2 receptor of the lungs thus downregulates its expression. This increases lung edema which leads to ALI/ARDS [16]. Cytokine storm- a large replication of the virus in respiratory epithelial cells equipped with by COVID-19 infection stimulate acute inflammation and severe respiratory disease. After infection into lung tissues, COVID-19 activates dendritic cells and macrophages that further produce pro-inflammatory cytokines such as IL12, IL-15, and IL-18. Interaction between these cytokines leads to the chemotaxis and activation of natural killer (NK) cells, Type 1 helper $\mathrm{T}$ (Th1) cells. They release granulocyte - macrophage colony - stimulating factor $(\mathrm{GM}-\mathrm{CSF})$ and interleukin - 6 (IL - 6), IFN- $\gamma$, TNF- $\alpha$, IL1 , IL-2 which results in a cytokine storm. Activated NK cells secrete granzymes, nitric oxide (NO), reactive oxygen species (ROS), perforin, and cytotoxic $\mathrm{T}$ lymphocytes to kill the virus. An excess amount of inflammatory molecules damages the epithelium and endothelium line and causing alveolar damage and limits gas exchange which causes pneumonia [16, 38].

Also, blood abnormalities, together with the low quantity of lymphocytes, hypoalbuminemia, high level of lactate dehydrogenase $(\mathrm{LDH})$, low level of neutrophils, C-reactive 
protein (CRP), and Angiotensin II were related to the severe lung injury [3]. When pulmonary fibrosis caused by COVID19 develops severe respiratory failure, professionals perform lung transplantation as a last step medication with strict precaution. But before LT several factors should be in concern- despite maximum medical support, irreversibility of refractory respiratory failure, positive-turned-negative virology, absence of other organ dysfunction, etc. [39].

\subsection{Effects on Heart}

A recent study has found that Covid-19 can cause multiorgan failure though it primarily affects lungs by causing interstitial pneumonitis [40]. Patients with hypertension, coronary heart disease, diabetes, kidney disease, and immunosuppressive diseases are said to be at higher risk for the mortality and morbidity of the disease. The elder people are at greater risk in terms of COVID-19 mortality comparing to the younger ones [17, 20, 41, 42]. Patients with pre-existing cardiovascular disease (especially hypertension) had the highest morbidity rate amongst the other complications. A recent study based on 5,000 patients in New York City, found that people with hypertension (57\%), obesity (40\%), and diabetes (34\%) were co-morbid [40]. Another analysis among 72,314 COVID-19 patients from the Chinese Centre for Disease Control and Preventions reported that $13 \%$ had Hypertension, 5\% had diabetes and $4 \%$ had known cardiovascular diseases. Among them, $40 \%$ of patients with hypertension, $20 \%$ of patients with diabetes, and $22 \%$ of patients with pre-existing cardiovascular diseases did not survive [41]. Different published reports from medRxiv, bioRxiv suggested the detailed evidence of myocardial injury in $20-40 \%$ of hospitalized patients such as cardiac pain, cardiac arrhythmias, coronary failure, and cardiac death [40]. Interestingly, it is also evident that COVID-19 may lead to heart disease even in people without having pre-existing cardiovascular diseases [20].

\subsubsection{Hypertension and COVID-19}

Though it is still not clear if hypertension works as a risk factor for COVID-19 or not. However, several studies and available data suggested that it is more prevalent in patients with severe conditions. A study among 44,672 patients in China CDC reported that hypertension was common in $12.8 \%$ and $39.7 \%$ were eventually died $[17,40]$. It is also reported that hypertension increased the odds ratio (OR) for death in COVID-19 patients and it is more frequent in older people and they have a higher mortality rate than younger people [40].

\subsubsection{Cardiac Arrhythmia and COVID-19}

Viral infections are said to be interlinked with myocardial inflammation, activation of the sympathetic nervous system, and metabolic dysfunction; together they lead to cardiac arrhythmia. A study on 138 COVID-19 patients in China revealed that $16.7 \%$ of patients developed an arrhythmia, the second most severe complication seen among COVID-19 patients [40]. It is also evident that ICU patients had a higher occurrence of arrhythmia comparing to non-ICU patients. Another study with 187 hospitalized patients stated that 5.9\% developed ventricular tachycardia or ventricular fibrillation [41]. The China CDC also reported that some COVID-19 patients showed symptoms like chest tightness, palpitations, etc. as well [40].

\subsubsection{Myocardial Injury, Heart Failure, and COVID-19}

Elevated cardiac troponin-I $(>28 \mathrm{pg} / \mathrm{L})$ in the serum is one of the main causes of cardiac injury [17]. Developing acute cardiac injury is very common amongst the COVID-19 patients as reported in different parts of the world [20]. Increased level of blood creatine kinase (CK), lactate dehydrogenase (LDH), cardiac troponin-I, myocardial dysfunction, or electrocardiographic abnormalities seems to be more common among COVID-19 patients [17, 20, 40, 41]. Elevated levels of troponin and inflammatory biomarkers, myoglobin, serum ferritin, CRP, and IL-6 were found in non-survivors of COVID-19 [17]. A recent study reported that approximately $20 \%$ of COVID-19 patients developed a cardiac injury, $10.6 \%$ had coronary heart disease, $5.3 \%$ had cardiovascular disease and $4.1 \%$ had heart failure [20]. A study in China among 191 patients from two hospitals revealed that $23 \%$ of corona survivors experienced heart failure and among the non-survivors, 52\% had developed heart failure $[40,41]$. In addition, several studies across the world showed similar consequences of COVID-19 patients developing a myocardial injury.

\subsubsection{Myocarditis and COVID-19}

Myocarditis is a well-recognized complication among patients with COVID-19 and it is one of the probable causes of acute cardiac injury. Myocarditis appeared in patients after a prolonged period (approximately 10-15 days) of the infection. Clinically COVID-19 myocarditis may lead to palpitations or mild chest discomfort, which is difficult to distinguish from other causes. Transient change in the ECG report, MRI report, and endomyocardial biopsy can detect myocarditis [40]. A 37 years old man with COVID-19 in China was admitted to the hospital with chest pain, diarrhea, and dyspnea and indicating myocarditis with acute elevation of cTnT and NT-proBNP. Coronavirus particles were found within cytopathic interstitial cells of the myocardium as well in the endomyocardial biopsy of a 69 years old woman with COVID-19 [41].

\subsubsection{Thrombosis and COVID-19}

COVID-19 hospitalized patients also showed altered coagulation parameters with remarkable elevation in D-dimer and fibrin degradation products in blood. In a cohort study, researchers found an elevated level of D-dimer and fibrin degradation products in non-survivors compared to the survivors. These differences became more apparent during daily measurements and by later stages, $71.4 \%$ of nonsurvivors showed disseminated intravascular coagulation [40, 41].

However, the actual mechanism of SARS-CoV-2 affecting the cardiac system is still unknown. Nevertheless, the 
presence of the ACE2 receptor as one of the key components of the renin-angiotensin system and elevated level of IL-6 may illustrate how COVID-19 affecting the cardiac system and thus leading to cardiovascular disease.

\subsection{Renal Injury}

Studies suggested that COVID-19 patients may develop acute kidney injury (AKI). In a report of 193 cases in China, $60 \%$ of cases had proteinuria, $48 \%$ cases had hematuria, and $20 \%$ showed elevated uric acid (UA), 31\% had a higher blood urea nitrogen (BUN) and $22 \%$ had a higher serum creatinine levels [43]. Interestingly, the prevalence of AKI has also been observed in the past coronavirus infections, SARS, and MERS [44]. Although the exact etiology of AKI progression is still uncertain, it is expected to be multifactorial related to both the direct and indirect impacts of the infection on the kidney $[45,46]$. The ACE2 receptor is abundantly expressed in proximal tubules as well as in podocytes, parietal epithelial cells, collecting ducts, and distal tubules of the kidney $[12,47]$. These segments may act as a potential route of entry for the SARS-CoV2 in kidney cells [12]. Besides, SARS-CoV2 presence in the urine of an infected individual also implies the possible renal tropism of the virus [48]. Besides, studies on histopathological analysis of kidneys demonstrate collapsing glomerulopathy, swelling of mitochondria and lysosome, vacuolar degeneration, acute tubular injury (ATI) with loss of luminal brush border, and thrombosis. Furthermore, coronavirus-like particles have been sorted in the renal proximal tubular epithelium as well as in podocytes, and expression of viral nucleoprotein (NP) antigen has been observed in the kidneys [49-53]. Interestingly, similar findings were found in previous coronavirus infections SARS and MERS $[54,55]$. Therefore, this evidence suggests the potential direct cytopathic impact of the virus which might lead to the progression of AKI [53].

Additionally, viral-induced cytokine storm may contribute to kidney impairment [56]. Proinflammatory cell infiltration (e.g., CD68+ macrophages, CD56+ natural killer (NK) cells, CD4 $+\mathrm{T}$ cells, and CD8+ $\mathrm{T}$ cells) have been identified in tubulointerstitium of infected tissue and hyperactivation of these cells may gradually induce epithelial cell apoptosis, fibrosis, and trigger microvasculature alteration. Furthermore, complement-mediated C5-B9 complex or membrane attack complex deposition in tubules has been observed and activation of these complexes triggers renal parenchymal cells to release pro-inflammatory cytokines, profibrotic factors, and ROS that leads to renal injury $[53,57]$.

The underlying acute respiratory failure (ARDS) syndrome is considered a risk factor for the development of AKI in seriously ill patients [51]. Interorgan cross-talk between lung and kidney may worsen renal performance through several potential mechanisms including hemodynamic instability, biotrauma, hypoxia [58, 59]. Increased levels of proinflammatory cytokines (e.g., IL-6, plasminogen activator inhibitor-1 (PAI-1), soluble TNF-I, and TNF-II receptors) released from injured lungs might contribute to the incidence of AKI in patients with ARDS [58]. Other plausible factors include ischemia, drug-induced nephrotoxicity, rhabdomyolysis may also contribute to damage to the kidney $[49,60,61]$. AKI amplifies the risk of mortality rate in COVID-19 patients, and hence proper surveillance of renal involvement and effective intervention may help to reduce the fatality [62].

\subsection{Neurological Manifestation}

Multiple contextual analyses indicate that SARS-CoV-2 has the ability to develop neurological symptoms [13, 22, 63-65]. Techniques like electron microscopy, immunohistochemistry, and real-time reverse transcription PCR have been used to detect the presence of the virus in the brain [63]. A case study of COVID-19 patients in Wuhan showed that about $36.4 \%$ of patients (78 out of 214) had experienced various neurological manifestations [66]. SARSCoV-2 has the potentiality of invading the CNS, like the other members of the coronavirus family [67]. Additionally, neurological injury at some stage in the course of SARSCoV-2 infection may be related to coagulopathy, with a higher rate of systemic and neighborhood thrombotic events [13]. Three possible pathways have been postulated till now from numerous researches that are used by SARS-CoV to invade the nervous system.

\subsubsection{Hematogenous Route}

After infecting the respiratory airways, $\mathrm{HCoV}$ could cross the basolateral surfaces of epithelial cells into the blood circulation, in a process called viremia [68]. The virus may invade CNS by infecting endothelial cells of the Blood-Brain Barrier (BBB), epithelial cells of the Blood-CSF Barrier, or by using inflammatory cells as Trojan Horse [69]. SARSCoV-2 may attach to the ACE2 receptor expressed by endothelial cells of the BBB [70]. Once the virus enters into vascular and neuronal tissue, it could begin the cycle of viral budding contacting ACE2 on neurons, glia, and vessels [18]. Circulating with bloodstream SARS-CoV-2 can also infect epithelial cells of the Blood-CSF barrier in the choroid plexus (CP), which is located in the ventricles of the brain [71]. Interestingly, several strains of coronavirus, including SARS, have shown the potentiality to infect numerous types of leukocytes both in vitro and in vivo, where leukocytes serve as a reservoir and vector for neuro-invasive $\mathrm{HCoV}[69$, 72-74]. This mechanism is termed "the Trojan Horse" mechanism, where the affected immune cells move from the blood and get through the BBB to infect the CNS. SARS virus can infect several immune cells, particularly the monocytes, macrophages, and T lymphocytes [72].

\subsubsection{Neuronal Retrograde Route}

The $\mathrm{HCoV}$ virus infects peripheral neurons, like olfactory receptor neurons, the particular trigeminal nerve that lies in the nasal cavity or sensory fibers of the vagus nerve in the brain stem, to invade the CNS by the axonal retrograde transport [69, 75, 76]. Evidence indicated that the virus attacks the CNS by synapses just after invading the peripheral nerve terminals [71]. Loss of sense of smell and 
loss of sense of taste illustrate the direct entry of SARS-CoV2 to the brain via the cribriform plate. SARS-CoV-2 may enter the CNS directly by the olfactory nerve which is likely another possible mechanism for the invasion $[18,70,71]$.

\subsubsection{Lymphatic Pathway}

Several members of the coronavirus family including SARS-CoV, can infect cells involved in intrinsic immunity and thus invade CNS. Besides, SARS-CoV can infect monocyte-derived dendritic cells (DCs). Infected DCs thus transport the virus to the lymphatic tissue, which can be a crucial route of infection [77].

Moreover, studies suggested that COVID-19 patients develop Acute Necrotizing Encephalopathy (ANE), which is associated with cytokine storm. Evidence also indicates that an elevated level of the plasma concentration of different cytokines in the initial stage of COVID-19 infection may also help the virus to invade the CNS $[3,78]$. However, further research is needed to comprehend the invading mechanism of COVID-19 to the CNS for better therapeutic development.

\subsection{Liver Injury}

Several studies suggested that patients with SARS and MERS had developed liver injury during the infection [79, 80]. Generally, coronavirus attacks the pulmonary system and creates problems such as mild respiratory disease to pneumonia but may lead to acute respiratory distress syndrome or multi-organ dysfunction in severe cases [81]. Though some reports support the fact that COVID-19 may cause liver dysfunction but the severity and mortality of this action are still unknown [82-84]. Besides, it is not yet clear whether the liver damage is done directly by viral inclusion or by inflammation response of the immune system [85].

In China, COVID-19 patients had experienced leukopenia, lymphocytopenia, and a higher level of CRP along with acute respiratory failure and severe hypoxemia. In most of these cases, patients were found with septic shock and multiple organ dysfunction. Interestingly, some liver autopsy showed hepatomegaly with dark red, hepatocyte degeneration accompanied by lobular focal necrosis and neutrophil infiltration, infiltration of lymphocytes, and monocytes in the portal area, and congestion of hepatic sinuses with microthrombosis [80].

From the analysis of single-cell sequencing and immunohistochemistry, it is evident that a high level of ACE2 was expressed in the biliary epithelial cells and a very low amount was measured in the hepatocytes [21, 80]. Interestingly, a higher level of ACE2 expression is measured in cholangiocytes rather than in endothelial cells, hepatocytes, and kupffer cells where the expression was minimum $[80,83]$. So, it can be predicted that the virus may get into the liver through the bile duct. But it is still unknown whether and how the virus is directly involved in the liver.

According to some studies, a high amount of liver markers such as alanine aminotransferase (ALT), aspartate aminotransferase (AST), gamma-glutamyl transferase (GGT), and bilirubin are common in critical patients rather than in mid-COVID-19 patients. In most of the cases, an elevated number of cytokines, including IL- 6 , IL- $1 \beta$, TNF- $\alpha$, and cytokine storm is also observed in the liver [82]. However, patients having pre-existing chronic hepatic diseases were found to have an increased level of key liver markers such as ALT, AST, GGT, and total bilirubin [82, 84, 86]. So, in both cases, patients were diagnosed with an elevated amount of liver markers.

The patients who have liver injuries are at higher risk because of the medicine used in the treatment of COVID-19, possible viral load in hepatic cells, hypoxia, and systematic inflammation. Drugs such as antibiotics, antiviral drugs, pain killers, etc., that are used in treating COVID-19 patients may damage the liver as these drugs can create hepatic toxicity [82].

Both the Innate and Adaptive immune system triggers the inflammatory responses and pulmonary injury to COVID-19 patients. Generally, in COVID-19 patients, CD4+ T-cells activate $\mathrm{B}$-cells and induce the production of virus-specific antibodies. The presence of a high level of CD8+ in the pulmonary interstitium may help to clear the coronavirus from the affected cells and reduce immune injury [80]. One study suggested that in COVID-19 patients lymphopenia and CRP levels independently damaged the liver where inflammatory cytokine storm played a major role [81].

Evidences suggested that the cytokine storms are associated with the adverse outcomes of COVID-19, thus leads to the continuous activation of lymphocytes and macrophages. These cells eventually then secret a high level of inflammatory cytokines. Typical lymphocytes during the infection may result in an elevated level of IL-2, IL-6, IL-10, and IFN- $\gamma$ and that might be the reason for liver dysfunction as well [81].

\section{Conclusion}

SARS-CoV-2 is mainly a respiratory virus but has the potentiality to infect several organs like the heart, liver, kidney, CNS, etc. The spike protein of SARS-COV-2 can recognize the "Angiotensin-Converting Enzyme 2 (ACE2)" receptor of the host cell, which is expressed in many organs in humans. The world is facing a COVID-19 pandemic, which requires a lot of research regarding the development of potential treatment of COVID-19, apparently a safe vaccine. There are more than 100 vaccine candidates enlisted by WHO which are under trial now. To combat the virus, an accurate mechanism of action, clinical features need to be established. Early prognosis of the multi-organ dysfunction following the onset of the disease could help to treat and manage patients effectively in hospital settings. There is emerging evidence of patients suffering from post-COVID19 multi-organ dysfunction and are requiring various replacement therapies. Further research should be conducted to explore the distinct pathophysiology of multi-organ dysfunction. Besides, the COVID-19 pandemic has made us realize the importance of co-operating between pharmaceuticals companies, governments, and regulatory 
agencies. Planning to survive this kind of pandemic should be organized before the occurrence.

\section{Author's Contribution Statement}

MAM conceived the idea and revised the manuscript. FS, $\mathrm{KZ}$, AS, and SF contributed to drafting the manuscript. All authors approved the final version of the manuscript.

\section{Conflict of Interest}

All the authors do not have any possible conflicts of interest.

\section{References}

[1] Morens D. M., Folkers G. K., and Fauci A. S. (2009). What is a pandemic? Journal of Infectious Diseases, 200: 1018-1021.

[2] Cucinotta D. and Vanelli M. (2020). WHO declares COVID19 a pandemic. Acta Biomedica, 91: 157-160.

[3] Saghazadeh A. and Rezaei N. (2020). Immune-epidemiological parameters of the novel coronavirus-a perspective. Expert Review of Clinical Immunology, 16: 465-470.

[4] Li Q., Guan X., Wu P., Wang X., Zhou L., Tong Y., et al. (2020). Early transmission dynamics in Wuhan, China, of novel coronavirus-infected pneumonia. New England Journal of Medicine, 382: 1199-1207.

[5] Devaux C. A., Rolain J. M., and Raoult D. (2020). ACE2 receptor polymorphism: Susceptibility to SARS-CoV-2, hypertension, multi-organ failure, and COVID-19 disease outcome. Journal of Microbiology, Immunology and Infection, 53 (3): 425-435.

[6] Yuefei J., Haiyan Y., Wangquan J., Weidong W., Shuaiyin C. W. Z., and Duan G. (2020). Virology, epidemiology, pathogenesis, and control of COVID-19. Viruses, 12 (4): 372.

[7] Wang C., Horby P. W., Hayden F. G., and Gao G. F. (2020). A novel coronavirus outbreak of global health concern. The Lancet, 395: 470-473.

[8] Hu B., Guo H., Zhou P., and Shi Z. L. (2020). Characteristics of SARS-CoV-2 and COVID-19. Nature Reviews Microbiology, 1-14.

[9] Zhang Y. Z. and Holmes E. C. (2020). A genomic perspective on the origin and emergence of SARS-CoV-2. Cell, 181: 223227.

[10] Peiris J. S. M., Guan Y., and Yuen K. Y. (2004). Severe acute respiratory syndrome. Nature Medicine, 10: S88-97.

[11] Chen L., Lou J., Bai Y., and Wang M. (2020). COVID-19 disease with positive fecal and negative pharyngeal and sputum viral tests. American Journal of Gastroenterology, 115: 790.

[12] Zou X., Chen K., Zou J., Han P., Hao J., and Han Z. (2020). Single-cell RNA-seq data analysis on the receptor ACE2 expression reveals the potential risk of different human organs vulnerable to 2019-nCoV infection. Frontiers of Medicine, e1-8.
[13] Huang C., Wang Y., Li X., Ren L., Zhao J., Hu Y., et al. (2020). Clinical features of patients infected with 2019 novel coronavirus in Wuhan, China. The Lancet, 395: 497-506.

[14] Napoleoni L. (2005). Profile of a killer. Foreign Policy, 151: 36-43.

[15] Adhikari S. P., Meng S., Wu Y., Mao Y., Ye R., Wang Q., et al. (2020). A scoping review of 2019 novel coronavirus during the early outbreak period: Epidemiology, causes, clinical manifestation and diagnosis, prevention and control. Research Square.

[16] Hu B., Huang S., and Yin L. (2020). The cytokine storm and COVID-19. Journal of Medical Virology.

[17] Madjid M., Safavi-Naeini P., and Solomon S. D., and Vardeny O. (2020). Potential effects of coronaviruses on the cardiovascular system: A Review. JAMA Cardiology, 5: 831-840.

[18] Baig A. M., Khaleeq A., Ali U., and Syeda H. (2020). Evidence of the COVID-19 Virus targeting the CNS: tissue distribution, host-virus interaction, and proposed neurotropic mechanisms. ACS Chemical Neuroscience, 11: 995-998.

[19] Channappanavar R. and Perlman S. (2017). Pathogenic human coronavirus infections: causes and consequences of cytokine storm and immunopathology. Seminars in Immunopathology, 39: 529-539.

[20] Paramasivam A., Priyadharsini J. V., Raghunandhakumar S., and Elumalai P. (2020). A novel COVID-19 and its effects on cardiovascular disease. Hypertension Research, 43: 729-730.

[21] Kumar M. P., Mishra S., Jha D. K., Shukla J., et al. (2020). Coronavirus disease (COVID-19) and the liver: a comprehensive systematic review and meta-analysis. Hepatology International, 14: 711-722.

[22] Arbour N., Day R., Newcombe J., and Talbot P. J. (2000). Neuroinvasion by human respiratory coronaviruses. Journal of Virology, 74: 8913-8921.

[23] Ioannidis J. P. A. (2020). Global perspective of COVID-19 epidemiology for a full-cycle pandemic. European journal of clinical investigation, e13421.

[24] Clark A., Jit M., Warren-Gash C., Guthrie B., et al. (2020). Global, regional, and national estimates of the population at increased risk of severe COVID-19 due to underlying health conditions in 2020: a modelling study. The Lancet Global Health, 8: 1003-1017.

[25] Burki T. (2020). China's successful control of COVID-19. The Lancet Infectious diseases, 3099: 19-20.

[26] Petersen E., Koopmans M., Go U., Hamer D, H., et al. (2020). Comparing SARS-CoV-2 with SARS-CoV and influenza pandemics. The Lancet Infectious Diseases, 20 (9): 238-244.

[27] Randolph H. E. and Barreiro L. B. (2020). Herd immunity: understanding COVID-19. Immunity, 52: 737-741.

[28] Conti P., Ronconi G., Caraffa A., Gallenga C. E., et al. (2020). Induction of pro-inflammatory cytokines (IL-1 and IL-6) and lung inflammation by Coronavirus-19 (COVI-19 or SARSCoV-2): anti-inflammatory strategies. Journal of biological regulators and homeostatic agents, 34: 327-331.

[29] Shi H., Han X., Jiang N., Cao Y., et al. (2020). Radiological findings from 81 patients with COVID-19 pneumonia in Wuhan, China: a descriptive study. The Lancet Infectious Diseases, 20: 425-434. 
[30] Zhao W., Zhong Z., Xie X., Yu Q., and Liu J. (2020). Relation between chest CT findings and clinical conditions of coronavirus disease (covid-19) pneumonia: A multicenter study. American Journal of Roentgenology, 214: 1072-1077.

[31] Pan F., Ye T., Sun P., Gui S., et al. (2020). Time course of lung changes on chest CT during recovery from 2019 novel coronavirus (COVID-19) pneumonia. Radiology, e200370.

[32] Inui, S., Fujikawa, A., Jitsu, M., Kunishima, N., et al. (2020). Chest CT findings in cases from the cruise ship "Diamond Princess" with coronavirus disease 2019 (COVID-19). Radiology. Cardiothoracic Imaging, 2 (2): e200110.

[33] McGonagle D., O’Donnell J. S., Sharif K., Emery P., and Bridgewood C. (2020). Immune mechanisms of pulmonary intravascular coagulopathy in COVID-19 pneumonia. The Lancet Rheumatology, 2: 437-445.

[34] Gattinoni L., Chiumello D., Caironi P., Busana M., et al. (2020). COVID-19 pneumonia: different respiratory treatments for different phenotypes? Intensive Care Medicine, 46: 1099-1102.

[35] Marini J. J. and Gattinoni L. (2020). Management of COVID19 respiratory distress. Jama, 7: 435-444.

[36] Menter T., Haslbauer J. D., Nienhold R., Savic S., Hopfer H., et al. (2020). Postmortem examination of COVID-19 patients reveals diffuse alveolar damage with severe capillary congestion and variegated findings in lungs and other organs suggesting vascular dysfunction. Histopathology, 77: 198-209.

[37] Carsana L., Sonzogni A., Nasr A., Rossi R. S., et al. (2020). Pulmonary post-mortem findings in a series of COVID-19 cases from northern Italy: a two-centre descriptive study. The Lancet Infectious Diseases, 20: 1135-1140.

[38] Pascarella G., Strumia A., Piliego C., Bruno F., et al. (2020). COVID-19 diagnosis and management: a comprehensive review. Journal of Internal Medicine, 288: 192-206.

[39] Chen J. Y., Qiao K., Liu F., Wu B., Xu X., et al. (2020). Lung transplantation as therapeutic option in acute respiratory distress syndrome for coronavirus disease 2019-related pulmonary fibrosis. Chinese Medical Journal, 133: 1390-6.

[40] Guzik T. J., Mohiddin S. A., Dimarco A., Patel V., Savvatis K., et al. (2020). COVID-19 and the cardiovascular system: Implications for risk assessment, diagnosis, and treatment options. Cardiovascular Research, 116: 1666-87.

[41] Lang J. P., Wang X., Moura F. A., Siddiqi H. K., Morrow D. A., and Bohula E. A. (2020). A current review of COVID-19 for the cardiovascular specialist. American Heart Journal, 226: 29-44.

[42] ALkharashi N. A. (2019). Brief communication. Saudi Medical Journal, 40: 1290-1293.

[43] Pei G., Zhang Z., Peng J., Liu L., Zhang C., Yu C., et al. (2020). Renal involvement and early prognosis in patients with COVID-19 pneumonia. Journal of the American Society of Nephrology, 31 (6): 1157-1165.

[44] Yin Y. and Wunderink R. G. (2018). MERS, SARS and other coronaviruses as causes of pneumonia. Respirology, 23 (2): 130-137.

[45] Ali N. and Mahmood S. (2020). Kidney Injury in COVID-19: an emerging concern to the clinician. SN Compr Clin Med, 2: 1808-1809.
[46] Naicker S., Yang C. W., Hwang S. J., Liu B. C., Chen J. H., and Jha V. (2020). The novel coronavirus 2019 epidemic and kidneys. Kidney International, 97 (5): 824-828.

[47] Hamming I., Timens W., Bulthuis M. L. C., Lely A. T., Navis G. J., and van Goor H. (2004). Tissue distribution of ACE2 protein, the functional receptor for SARS coronavirus. A first step in understanding SARS pathogenesis. Journal of Pathology, 203 (2): 631-637.

[48] Sun J., Zhu A., Li H., Zheng K., Zhuang Z., et al. (2020). Isolation of infectious SARS-CoV-2 from urine of a COVID19 patient. Emerging Microbes and Infections, 9 (1): 991-993.

[49] Su H., Yang M., Wan C., Yi L. X., Tang F., et al. (2020). Renal histopathological analysis of 26 postmortem findings of patients with COVID-19 in China. Kidney International. 98 (1): 219-227.

[50] Kissling S., Rotman S., Gerber C., Halfon M., Lamoth F., et al (2020). Collapsing glomerulopathy in a COVID-19 patient. Kidney International, 98 (1): 228-231.

[51] Santoriello D, Khairallah P, Bomback AS, Xu K, Kudose S, Batal I, et al. (2020). Postmortem kidney pathology findings in patients with COVID-19. Journal of the American Society of Nephrology, 31 (9): 2158-2167.

[52] Kudose S., Batal I., Santoriello D., Xu K., Barasch J., et al. (2020). Kidney biopsy findings in patients with COVID-19. Journal of the American Society of Nephrology, 31 (9): 19591968.

[53] Diao B., Wang C., Wang R., Feng Z., Tan Y., et al. (2020). Human kidney is a target for novel severe acute respiratory syndrome coronavirus 2 (SARS-CoV-2) Infection. MedRxiv.

[54] Ding Y., He L., Zhang Q., Huang Z., Che X., Hou J., et al. (2004). Organ distribution of severe acute respiratory syndrome (SARS) associated coronavirus (SARS-CoV) in SARS patients: Implications for pathogenesis virus transmission pathways. Journal of Pathology, 203 (2): 622-630.

[55] Yeung M. L., Yao Y., Jia L., Chan J. F. W., Chan K. H., et al. (2016). MERS coronavirus induces apoptosis in kidney and lung by upregulating Smad7 and FGF2. Nature Microbiology, $1(3): 1-8$

[56] Ronco C. and Reis T. (2020). Kidney involvement in COVID19 and rationale for extracorporeal therapies. Nature Reviews Nephrology, 16: 308-310.

[57] Khouchlaa A. and Bouyahya A. (2020). COVID-19 nephropathy; probable mechanisms of kidney failure. Journal of Nephropathology, 9 (4): 1-4.

[58] Joannidis M., Forni L. G., Klein S. J., Honore P. M., Kashani K., et al. (2020). Lung-kidney interactions in critically ill patients: consensus report of the acute disease quality initiative (ADQI) 21 workgroup. Intensive Care Medicine, e1-19.

[59] Husain S. F., Slutsky A. S., and Ronco C. (2016). Lungkidney cross-talk in the critically ill patient. American Journal of Respiratory and Critical Care Medicine, 194 (4): 402-414.

[60] Oussalah A., Gleye S., Clerc Urmes I., Laugel E., Callet J., et al. (2020). Long-term ACE inhibitor/ARB use is associated with severe renal dysfunction and acute kidney injury in patients with severe COVID-19: Results from a referral center cohort in the Northeast of France. Clinical Infectious Diseases, e1-10. 
[61] Sabbahy M. E. and Vaidya V. S. (2011). Ischemic kidney injury and mechanisms of tissue repair. Wiley Interdisciplinary Reviews: Systems Biology and Medicine, 3 (5): 606-618.

[62] Cheng Y., Luo R., Wang K., Zhang M., Wang Z., Dong L., et al. (2020). Kidney disease is associated with in-hospital death of patients with COVID-19. Kidney International, 97 (5): 829838 .

[63] Netland J., Meyerholz D. K., Moore S., Cassell M., and Perlman S. (2008). Severe acute respiratory syndrome coronavirus infection causes neuronal death in the absence of Encephalitis in mice transgenic for human ACE2. Journal of Virology, 82: 7264-7275.

[64] Alper G. (2012). Detection of coronavirus in the central nervous system of a child with acute disseminated Encephalomyelitis. Journal of Child Neurology, 27: 14081425 .

[65] Guastalegname M. and Vallone A. (2020). Self-reported olfactory and taste disorders in patients with severe acute respiratory coronavirus 2 infection: A cross-sectional study. Clinical Infectious Diseases, 71 (15): 888-890.

[66] Mao L., Jin H., Wang M., Hu Y., Chen S., He Q., et al. (2020). Neurologic manifestations of hospitalized patients with coronavirus disease 2019 in Wuhan, China. JAMA Neurology, 77: 683-690.

[67] Aghagoli G., Gallo Marin B., Katchur N. J., Chaves-Sell F., Asaad W. F., and Murphy S. A. (2020). Neurological involvement in COVID-19 and potential mechanisms: A review. Neurocritical Care, e1-10.

[68] Dijkman R., Jebbink M. F., Koekkoek S. M., Deijs M., et al. (2013). Isolation and characterization of current human coronavirus strains in primary human epithelial cell cultures reveal differences in target cell tropism. Journal of Virology, 87: 6081-6090.

[69] Desforges M., Le C. A., Dubeau P., Bourgouin A., Lajoie L., Dubé M., et al. (2019). Human coronaviruses and other respiratory viruses: Underestimated opportunistic pathogens of the central nervous system? Viruses, 12: 1-28.

[70] Zubair A. S., McAlpine L. S., Gardin T., Farhadian S., Kuruvilla D. E., and Spudich S. (2020). Neuropathogenesis and neurologic manifestations of the coronaviruses in the age of coronavirus disease 2019: A review. JAMA Neurology, 77 (8): 1018-1027.

[71] Iroegbu J. D., Ifenatuoha C. W., and Ijomone O. M. (2020). Potential neurological impact of coronaviruses: implications for the novel SARS-CoV-2. Neurological Sciences, 41: 13291337.

[72] Gu J., Gong E., Zhang B., Zheng J., Gao Z., et al. (2005). Multiple organ infection and the pathogenesis of SARS. Journal of Experimental Medicine, 202: 415-424.
[73] Swanson P. and McGavern D. (2015). Portals of viral entry into the central nervous system. The Blood-Brain Barrier in Health and Disease, 2: 23-47.

[74] Desforges M., Miletti T. C., Gagnon M., and Talbot P. J. (2007). Activation of human monocytes after infection by human coronavirus 229E. Virus Research, 130: 228-240.

[75] Morris M. and Zohrabian V. M. (2020). Neuroradiologists, be mindful of the neuroinvasive potential of COVID-19. American Journal of Neuroradiology, 41: 37-39.

[76] Desforges M., Le C. A., Stodola J. K., Meessen-Pinard M., and Talbot P. J. (2014). Human coronaviruses: Viral and cellular factors involved in neuroinvasiveness and neuropathogenesis. Virus Research, 194: 145-158.

[77] Spiegel M., Schneider K., Weber F., Weidmann M., and Hufert F. T. (2006). Interaction of severe acute respiratory syndromeassociated coronavirus with dendritic cells. Journal of General Virology, 87: 1953-1960.

[78] Poyiadji N., Shahin G., Noujaim D., Stone M., Patel S., and Griffith B. (2020). COVID-19-associated acute hemorrhagic necrotizing encephalopathy: Imaging features. Radiology, 296: 119-120.

[79] De Haan C. A., De Wit M., Kuo, L., et al. (2003). The glycosylation status of the murine hepatitis coronavirus $M$ protein affects the interferogenic capacity of the virus in vitro and its ability to replicate in the liver but not the brain. Virology, 312 (2): 395-406.

[80] Li J., and Fan J. (2020). Characteristics and mechanism of liver injury in 2019 coronavirus disease. Journal of Clinical and Translational Hepatology, 8 (1): 13-17.

[81] Musa S. (2020). Hepatic and gastrointestinal involvement in coronavirus disease 2019 (COVID-19): What do we know till now? Arab Journal of Gastroenterology, 21: 3-8.

[82] Ali N. and Hossain K. (2020). Liver injury in severe COVID19 infection: current insights and challenges liver injury in severe COVID-19 infection: current insights and challenges. Expert Review of Gastroenterology \& Hepatology, 14 (10): 879-884.

[83] Zhang C., Shi L., and Wang F. (2020). Liver injury in COVID19: management and challenges. The Lancet Gastroenterology \& Hepatology, 5: 428-430.

[84] Jothimani D., Venugopal R., Abedin M. F., Kaliamoorthy I., and Rela M. (2020). COVID-19 and the liver. Journal of Hepatology, 73: 1231-1240.

[85] Ali N. (2020). Relationship between COVID-19 infection and liver injury: A review of recent data. Front Med, 7: 458.

[86] Ji D., Zhang D., Yang T., Mu J., Zhao P., Xu J., et al. (2020). Effect of COVID-19 on patients with compensated chronic liver diseases. Hepatology International, 14: 701-710. 